\title{
EXOTIC SMOOTH STRUCTURES ON 4-MANIFOLDS WITH ZERO SIGNATURE
}

\author{
ANAR AKHMEDOV AND B. DOUG PARK
}

\begin{abstract}
For every integer $k \geq 2$, we construct infinite families of mutually nondiffeomorphic irreducible smooth structures on the topological 4-manifolds $(2 k-1)\left(S^{2} \times S^{2}\right)$ and $(2 k-1)\left(\mathbb{C P}^{2} \# \overline{\mathbb{C P}}^{2}\right)$, the connected sums of $2 k-1$ copies of $S^{2} \times S^{2}$ and $\mathbb{C P}^{2} \# \overline{\mathbb{C P}}^{2}$.
\end{abstract}

\section{INTRODUCTION}

Let $M$ denote a closed smooth 4-manifold. Given an integer $k \geq 1$, let $k M$ denote the connected sum of $k$ copies of $M$. Let $\mathbb{C P}^{2}$ denote the complex projective plane and let $\overline{\mathbb{C P}}^{2}$ denote the underlying smooth 4-manifold $\mathbb{C P}^{2}$ equipped with the opposite orientation. Let $\mathbb{C P}^{2} \# \overline{\mathbb{P P}}^{2}$ denote the connected sum of $\mathbb{C P}^{2}$ and $\overline{\mathbb{C P}}^{2}$. To state our results, it will be convenient to introduce the following terminology.

Definition 1. We say that a 4 -manifold $M$ has $\infty^{2}$-property if there exist infinitely many mutually nondiffeomorphic irreducible symplectic 4-manifolds and infinitely many mutually nondiffeomorphic irreducible nonsymplectic 4-manifolds, all of which are homeomorphic to $M$.

The main goal of this paper is to prove the following.

Theorem 2. Both $(2 k-1)\left(S^{2} \times S^{2}\right)$ and $(2 k-1)\left(\mathbb{C P}^{2} \# \overline{\mathbb{C P}}^{2}\right)$ have $\infty^{2}$-property for every integer $k \geq 2$.

It was already proved in 3 , 2, that $(2 k-1)\left(S^{2} \times S^{2}\right)$ and $(2 k-1)\left(\mathbb{C P}^{2} \# \overline{\mathbb{C P}}^{2}\right)$ have $\infty^{2}$-property when $k \geq 138$ and $k \geq 25$, respectively. At the moment, it is unknown to the authors whether there is any overlap between the 4-manifolds constructed in [3, 2] and in this paper. The proof of Theorem 2 is given in Sections 2 4 . Our strategy is to apply the 'reverse engineering' technique of [7] to a suitably chosen nontrivial genus 2 surface bundle over a genus $k+1$ surface.

\section{Model COmplex SuRfaces}

Let $\Sigma_{g}$ denote a closed genus $g$ Riemann surface. For every integer $k \geq 1$, there is a free $\mathbb{Z} / 2$ action on a genus $2 k+1$ surface, $\tau_{k+1}: \Sigma_{2 k+1} \rightarrow \Sigma_{2 k+1}$, by rotating 180 degrees about the 'middle' $(k+1)$-th hole, such that $\Sigma_{2 k+1} /\left\langle\tau_{k+1}\right\rangle=\Sigma_{k+1}$. When $k=1$, we just get $\tau_{2}$ in [5]. Let $\tau_{1}$ be the elliptic involution on $\Sigma_{2}$ in Section 2 of [5]. There exists a free $\mathbb{Z} / 2$ action $\left(\tau_{1}, \tau_{k+1}\right)$ on the product $\Sigma_{2} \times \Sigma_{2 k+1}$ for every integer $k \geq 1$. Let $X_{k}$ denote the quotient space and let $q: \Sigma_{2} \times \Sigma_{2 k+1} \rightarrow X_{k}$ denote the quotient map. $X_{k}$ is a minimal complex surface of general type (cf. [10]), which is the total space of a nontrivial genus 2 surface bundle over a genus $k+1$ surface. We

Date: March 26, 2010. Revised on June 3, 2010.

2010 Mathematics Subject Classification. Primary 57R55; Secondary 57R17, 57R57. 
have $e\left(X_{k}\right)=e\left(\Sigma_{2} \times \Sigma_{2 k+1}\right) / 2=(-2)(-4 k) / 2=4 k, \sigma\left(X_{k}\right)=0, b_{1}\left(X_{k}\right)=2 k+4$ and $b_{2}\left(X_{k}\right)=8 k+6$.

Let $\left\{a_{1}, b_{1}, a_{2}, b_{2}\right\}$ and $\left\{c_{1}, d_{1}, \ldots, c_{2 k+1}, d_{2 k+1}\right\}$ be the set of simple closed curves representing the standard generators of $\pi_{1}\left(\Sigma_{2}, z_{0}\right)$ and $\pi_{1}\left(\Sigma_{2 k+1}, w_{0}\right)$, respectively. For the sake of brevity, we will usually abuse notation and write $a_{1}=q\left(a_{1} \times\left\{w_{0}\right\}\right)$ and $c_{1}=q\left(\left\{z_{0}\right\} \times c_{1}\right)$, etc. The next lemma is the analogue of Lemmas 5 and 6 in [5].

Lemma 3. The following $2 k+4$ loops generate $\pi_{1}\left(X_{k}\right)$ :

$$
a_{1}, b_{1}, c_{1}, d_{1}, \ldots, c_{k}, d_{k}, \tilde{c}_{k+1}, d_{k+1} .
$$

Moreover, the loops $c_{1}, d_{1}, \ldots, c_{k}, d_{k}, \tilde{c}_{k+1}, d_{k+1}$ represent elements of infinite order in $\pi_{1}\left(X_{k}\right)$.

Proof. As in [5], we can verify that

$$
\begin{gathered}
a_{2}=\tilde{c}_{k+1}^{-1} a_{1} \tilde{c}_{k+1}, \quad b_{2}=\tilde{c}_{k+1}^{-1} b_{1} \tilde{c}_{k+1}, \\
c_{2 k+2-j}=\tilde{c}_{k+1}^{-1} c_{j} \tilde{c}_{k+1}, \quad d_{2 k+2-j}=\tilde{c}_{k+1}^{-1} d_{j} \tilde{c}_{k+1},
\end{gathered}
$$

where $j=1,2, \ldots, k$. The bundle projection map

$$
X_{k}=\frac{\Sigma_{2} \times \Sigma_{2 k+1}}{\mathbb{Z} / 2} \longrightarrow \frac{\Sigma_{2 k+1}}{\mathbb{Z} / 2}=\Sigma_{k+1}
$$

maps $c_{1}, d_{1}, \ldots, c_{k}, d_{k}, \tilde{c}_{k+1}, d_{k+1}$ to the standard generators of $\pi_{1}\left(\Sigma_{k+1}\right)$.

The intersection form of $X_{k}$ is given by $(4 k+3) H$, where

$$
H=\left[\begin{array}{ll}
0 & 1 \\
1 & 0
\end{array}\right]
$$

A basis for the intersection form of $X_{k}$ is given by the following $4 k+3$ geometrically dual pairs:

$$
\begin{array}{cc}
\left(\left[a_{1} \times c_{1}\right],-\left[b_{1} \times d_{1}\right]\right), & \left(\left[a_{1} \times d_{1}\right],\left[b_{1} \times c_{1}\right]\right), \\
\left(\left[a_{2} \times c_{1}\right],-\left[b_{2} \times d_{1}\right]\right), & \left(\left[a_{2} \times d_{1}\right],\left[b_{2} \times c_{1}\right]\right), \\
\vdots & \vdots \\
\left(\left[a_{1} \times c_{k}\right],-\left[b_{1} \times d_{k}\right]\right), & \left(\left[a_{1} \times d_{k}\right],\left[b_{1} \times c_{k}\right]\right), \\
\left(\left[a_{2} \times c_{k}\right],-\left[b_{2} \times d_{k}\right]\right), & \left(\left[a_{2} \times d_{k}\right],\left[b_{2} \times c_{k}\right]\right), \\
\left(\left[\left(\tilde{a}_{1} \tilde{a}_{2}\right) \times \tilde{c}_{k+1}\right],-\left[b_{1} \times d_{k+1}\right]\right), & \left(\left[a_{1} \times d_{k+1}\right],\left[\left(\tilde{b}_{1} \tilde{b}_{2}\right) \times \tilde{c}_{k+1}\right]\right), \\
\left(\left[\Sigma_{2} \times\left\{w_{0}\right\}\right],\left[\left\{z_{0}\right\} \times \Sigma_{2 k+1}\right]\right) . &
\end{array}
$$

Here, $[\cdot]$ denotes the homology class of the image $q(\cdot)$ in the quotient manifold $X_{k}$ for short. All throughout, the $\tilde{c}_{k+1}$ path plays the role of $\tilde{c}_{2}$ in [5].

$$
\text { 3. Eхотіс }(2 k-1)\left(S^{2} \times S^{2}\right)
$$

By performing appropriate $2 k+4$ torus surgeries on $X_{k}$, we obtain exotic smooth structures on $(2 k-1)\left(S^{2} \times S^{2}\right)$. For example, we can choose to perform the following 
torus surgeries:

$$
\begin{array}{cc}
\left(a_{1}^{\prime} \times c_{1}^{\prime}, a_{1}^{\prime},-1\right), & \left(b_{1}^{\prime} \times c_{1}^{\prime \prime}, b_{1}^{\prime},-1\right), \\
\left(a_{2}^{\prime} \times c_{1}^{\prime}, c_{1}^{\prime},-1\right), & \left(a_{2}^{\prime \prime} \times d_{1}^{\prime}, d_{1}^{\prime},-n\right), \\
\left(a_{2}^{\prime} \times c_{2}^{\prime}, c_{2}^{\prime},-1 / p\right), & \left(a_{2}^{\prime \prime} \times d_{2}^{\prime}, d_{2}^{\prime},-1 / r\right), \\
\left(a_{2}^{\prime} \times c_{3}^{\prime}, c_{3}^{\prime},-1\right), & \left(a_{2}^{\prime \prime} \times d_{3}^{\prime}, d_{3}^{\prime},-1\right), \\
\vdots & \vdots \\
\left(a_{2}^{\prime} \times c_{k}^{\prime}, c_{k}^{\prime},-1\right), & \left(a_{2}^{\prime \prime} \times d_{k}^{\prime}, d_{k}^{\prime},-1\right), \\
\left(b_{1}^{\prime \prime} \times d_{k+1}^{\prime}, d_{k+1}^{\prime},-1\right), & \left(\left(\tilde{b}_{1} \tilde{b}_{2}\right) \times \tilde{c}_{k+1}^{\prime}, \tilde{c}_{k+1}^{\prime},-1\right),
\end{array}
$$

where $k, n, p, r$ are integers satisfying

$$
k \geq 1, \quad n \geq 1, \quad p \geq 0 \text { and } r \geq 0 .
$$

The prime and double prime notations are explained in [7]. The fourth, the fifth and the sixth surgeries are Luttinger surgeries (cf. 9, 6]) when $n=1, p \geq 1$ and $r \geq 1$, respectively.

Let $M_{k, n}^{p, r}$ denote the resulting closed 4-manifold. When $n=1, M_{k, 1}^{p, r}$ is symplectic for every triple $k, p, q$ satisfying (3). When $k=1$, there are no $-1 / p$ and $-1 / r$ surgeries, and we just get $M_{n}^{1}$ in [5]. Using exactly the same argument as in [5], we can prove the following.

Lemma 4. $\pi_{1}\left(M_{k, n}^{p, r}\right)$ is generated by

$$
a_{1}, b_{1}, a_{2}, b_{2}, c_{1}, d_{1}, \ldots, c_{k}, d_{k}, \tilde{c}_{k+1}, d_{k+1} .
$$

If $k \geq 2$, then the following relations hold in $\pi_{1}\left(M_{k, n}^{p, r}\right)$ :

$$
\begin{gathered}
a_{2}=\tilde{c}_{k+1}^{-1} a_{1} \tilde{c}_{k+1}, \quad b_{2}=\tilde{c}_{k+1}^{-1} b_{1} \tilde{c}_{k+1}, \quad b_{1}=\tilde{c}_{k+1}^{-1} b_{2} \tilde{c}_{k+1}, \\
{\left[b_{2}, d_{k+1}\right]=1, \quad\left[a_{1}^{-1} b_{1}^{-1} a_{2}, d_{k+1}\right]=1, \quad\left[a_{2}^{-1} b_{2}^{-1} a_{1}, d_{k+1}\right]=1,} \\
{\left[b_{1}^{-1}, d_{1}^{-1}\right]=a_{1}, \quad\left[a_{1}^{-1}, d_{1}\right]=b_{1}, \quad\left[b_{2}^{-1}, d_{1}^{-1}\right]=c_{1}, \quad\left[b_{2}, c_{1}^{-1}\right]^{n}=d_{1},} \\
{\left[b_{2}^{-1}, d_{2}^{-1}\right]=c_{2}^{p}, \quad\left[b_{2}, c_{2}^{-1}\right]=d_{2}^{r}, \ldots, \quad\left[b_{2}^{-1}, d_{k}^{-1}\right]=c_{k}, \quad\left[b_{2}, c_{k}^{-1}\right]=d_{k},} \\
\tilde{c}_{k+1}^{-1} a_{1} a_{2} \tilde{c}_{k+1} a_{1}^{-1} a_{2}^{-1}=d_{k+1}, \quad\left[a_{1}, d_{k+1}^{-1}\right]=\tilde{c}_{k+1}, \\
{\left[a_{1}, c_{1}\right]=1, \quad\left[b_{1}, c_{1}\right]=1, \quad\left[a_{2}, c_{1}\right]=1, \quad\left[a_{2}, d_{1}\right]=1, \quad\left[b_{1}, d_{k+1}\right]=1,} \\
{\left[a_{2}, c_{2}\right]=1, \quad\left[a_{2}, d_{2}\right]=1, \quad \ldots, \quad\left[a_{2}, c_{k}\right]=1, \quad\left[a_{2}, d_{k}\right]=1,} \\
{\left[a_{1}, c_{2}\right]=1, \quad\left[b_{1}, d_{2}\right]=1, \quad\left[a_{1}, d_{2}\right]=1, \quad\left[b_{1}, c_{2}\right]=1,} \\
\vdots \quad \vdots \quad[\quad \\
\left.\vdots a_{1}, c_{k}\right]=1, \quad\left[b_{1}, d_{k}\right]=1, \quad\left[a_{1}, d_{k}\right]=1, \quad\left[b_{1}, c_{k}\right]=1, \\
{\left[a_{1}, b_{1}\right]\left[a_{2}, b_{2}\right]=1, \quad\left[c_{1}, d_{1}\right] \cdots\left[c_{k}, d_{k}\right]\left[\tilde{c}_{k+1}, d_{k+1}\right]=1 .}
\end{gathered}
$$

Lemma 5. If $k \geq 2$, then $\pi_{1}\left(M_{k, n}^{p, r}\right) \cong \mathbb{Z} / p \oplus \mathbb{Z} / r$. In particular, $\pi_{1}\left(M_{k, n}^{1,1}\right)=0$ for every pair of integers $k \geq 2$ and $n \geq 1$.

Proof. By arguing exactly the same way as in the proof of Theorem 9 in [5], with $\tilde{c}_{k+1}$ and $d_{k+1}$ playing the roles of $\tilde{c}_{2}$ and $d_{2}$ in [5], respectively, we can show that $\left[b_{1}, b_{2}\right]=1$ and then $a_{1}=1$. From $a_{1}=1$, we can easily deduce that all other generators are trivial except for $c_{2}$ and $d_{2}$. Since

$$
\left[c_{2}, d_{2}\right]=\left[c_{1}, d_{1}\right] \cdots\left[c_{k}, d_{k}\right]\left[\tilde{c}_{k+1}, d_{k+1}\right]=1,
$$


the remaining generators $c_{2}$ and $d_{2}$ commute. From Lemma 3, we deduce that $c_{2}$ and $d_{2}$ have infinite order before the surgeries (2). We can now conclude that $\pi_{1}\left(M_{k, n}^{p, r}\right)$ is abelian and is isomorphic to $\mathbb{Z} / p \oplus \mathbb{Z} / r$.

For the remainder of this section, let $p=r=1$ and $k \geq 2$. The intersection form of $M_{k, n}^{1,1}$ is isomorphic to $(2 k-1) H$ (see (1D) with a basis given by

$$
\begin{array}{cc}
\left(\left[a_{1} \times c_{2}\right],-\left[b_{1} \times d_{2}\right]\right), & \left(\left[a_{1} \times d_{2}\right],\left[b_{1} \times c_{2}\right]\right), \\
\vdots & \vdots \\
\left(\left[a_{1} \times c_{k}\right],-\left[b_{1} \times d_{k}\right]\right), & \left(\left[a_{1} \times d_{k}\right],\left[b_{1} \times c_{k}\right]\right), \\
\left(\left[\Sigma_{2} \times\left\{w_{0}\right\}\right],\left[\left\{z_{0}\right\} \times \Sigma_{2 k+1}\right]\right) . &
\end{array}
$$

Hence the simply connected 4-manifolds $\left\{M_{k, n}^{1,1} \mid n \geq 1\right\}$ are all homeomorphic to $(2 k-1)\left(S^{2} \times S^{2}\right)$ by Freedman's theorem in 8 .

Let $Z_{k}$ denote the spin symplectic 4-manifold obtained from $X_{k}$ by performing $2 k+3$ Luttinger surgeries in (2) with $p=r=1$, but not $\left(a_{2}^{\prime \prime} \times d_{1}^{\prime}, d_{1}^{\prime},-n\right)$ surgery. In other words, $M_{k, n}^{1,1}$ is obtained from $Z_{k}$ by performing $\left(a_{2}^{\prime \prime} \times d_{1}^{\prime}, d_{1}^{\prime},-n\right)$ surgery. We have $e\left(Z_{k}\right)=4 k, \sigma\left(Z_{k}\right)=0, b_{1}\left(Z_{k}\right)=1, b_{2}\left(Z_{k}\right)=4 k$, and $b_{2}^{+}\left(Z_{k}\right)=2 k$. Let $A$ and $B$ denote the 2-dimensional cohomology classes of either $Z_{k}$ or $M_{k, n}^{1,1}$ that are Poincaré dual to the homology classes of $q\left(\Sigma_{2} \times\left\{w_{0}\right\}\right)$ and $q\left(\left\{z_{0}\right\} \times \Sigma_{2 k+1}\right)$, respectively.

If $S W_{Z_{k}}(L) \neq 0$, then by the adjunction inequality, $L=s A+t B$, where $s$ and $t$ are even integers satisfying $|s| \leq 2 k$ and $|t| \leq 2$. Since the dimension of the Seiberg-Witten moduli space for $L$ has to be nonnegative,

$$
L^{2}=2 s t \geq 2 e\left(Z_{k}\right)+3 \sigma\left(Z_{k}\right)=8 k .
$$

Hence $s= \pm 2 k, t= \pm 2, L= \pm(2 k A+2 B)=\mp c_{1}\left(Z_{k}\right)$, and by Taubes's theorem in 13 ,

$$
\left|S W_{Z_{k}}( \pm(2 k A+2 B))\right|=1 .
$$

It now follows from [7, 1] that $S W_{M_{k, n}^{1,1}}(L) \neq 0$ only when $L= \pm(2 k A+2 B)$, and

$$
\left|S W_{M_{k, n}^{1,1}}( \pm(2 k A+2 B))\right|=n .
$$

We conclude that $M_{k, n}^{1,1}$ 's are mutually nondiffeomorphic. By Taubes's theorem in [13, $M_{k, n}^{1,1}$ is nonsymplectic if $n \geq 2$.

\section{Exотіс $(2 k-1)\left(\mathbb{C P}^{2} \# \overline{\mathbb{C P}}^{2}\right)$}

Throughout this section, let $k \geq 2$ be an integer. Given an integer $m \geq 2$, let $M_{k, n}^{1,1}(m)$ denote the result of performing an $m$-surgery in $M_{k, n}^{1,1}$ along one of the tori in (5), say

$$
\left(a_{1}^{\prime} \times c_{2}^{\prime}, c_{2}^{\prime},+m\right) .
$$

By convention, we define $M_{k, n}^{1,1}(1)=M_{k, n}^{1,1}$.

Lemma 6. If $k \geq 2$, then $\pi_{1}\left(M_{k, n}^{1,1} \backslash q\left(a_{1}^{\prime} \times c_{2}^{\prime}\right)\right)=0$.

Proof. The $q\left(a_{1}^{\prime} \times c_{2}^{\prime}\right)$ torus intersects the $q\left(b_{1} \times d_{2}\right)$ torus negatively once in $M_{k, n}^{1,1}$. Hence any meridian of the $q\left(a_{1}^{\prime} \times c_{2}^{\prime}\right)$ torus will be a conjugate of $\left[b_{1}, d_{2}\right]^{ \pm 1}$. It follows that $\pi_{1}\left(M_{k, n}^{1,1} \backslash q\left(a_{1}^{\prime} \times c_{2}^{\prime}\right)\right)$ is normally generated by the generators listed in (44). Note 
that all the relations in Lemma 4 continue to hold in $\pi_{1}\left(M_{k, n}^{1,1} \backslash q\left(a_{1}^{\prime} \times c_{2}^{\prime}\right)\right)$, except possibly for the relation $\left[b_{1}, d_{2}\right]=1$. In the proof of Lemma 5 with $p=r=1$, we were able to kill all generators in (44) without making use of the relation $\left[b_{1}, d_{2}\right]=1$. Hence the generators in (4) are still trivial in $\pi_{1}\left(M_{k, n}^{1,1} \backslash q\left(a_{1}^{\prime} \times c_{2}^{\prime}\right)\right)$ and our lemma follows.

Since $\pi_{1}\left(M_{k, n}^{1,1} \backslash q\left(a_{1}^{\prime} \times c_{2}^{\prime}\right)\right)=0$, we can interpret (6) as a 'generalized logarithmic transformation' of multiplicity $m$. When $n=1$, we can perturb the symplectic form on $M_{k, 1}^{1,1}$ such that $q\left(a_{1}^{\prime} \times c_{2}^{\prime}\right)$ becomes a symplectic submanifold. Hence our generalized logarithmic transformation can be performed symplectically (cf. [12]), and the resulting 4-manifold $M_{k, 1}^{1,1}(m)$ is symplectic. Note that $\pi_{1}\left(M_{k, n}^{1,1}(m)\right)=0$, $e\left(M_{k, n}^{1,1}(m)\right)=4 k, \sigma\left(M_{k, n}^{1,1}(m)\right)=0$, and $b_{2}^{+}\left(M_{k, n}^{1,1}(m)\right)=2 k-1$ for every triple of integers $k \geq 2, n \geq 1$, and $m \geq 1$. that

Since $\pi_{1}\left(M_{k, n}^{1,1} \backslash q\left(a_{1}^{\prime} \times c_{2}^{\prime}\right)\right)=0$, Corollary 21 in [11] applies and we conclude

$$
S W_{M_{k, n}^{1,1}(m)}(L)= \begin{cases}n & \text { if } L= \pm(2 k A+2 B)+j T \\ 0 & \text { otherwise }\end{cases}
$$

where $j \in\{-(m-1),-(m-3), \ldots, m-3, m-1\}$, and $T$ is the cohomology class of $M_{k, n}^{1,1}(m)$ that is Poincare dual to the core torus of the logarithmic transformation.

Since every Seiberg-Witten basic class is characteristic, we must have

$$
w_{2}\left(M_{k, n}^{1,1}(m)\right) \equiv \pm(2 k A+2 B)+(m-1) T \equiv(m-1) T \quad(\bmod 2) .
$$

Hence we conclude that

$$
w_{2}\left(M_{k, n}^{1,1}(m)\right) \equiv \begin{cases}0 & \text { if } m \text { is odd } \\ T & \text { if } m \text { is even. }\end{cases}
$$

Since $T$ is primitive, $T \not \equiv 0(\bmod 2)$. It follows that $M_{k, n}^{1,1}(m)$ is spin if $m$ is odd and nonspin if $m$ is even. By Freedman's theorem in [8], $M_{k, n}^{1,1}(m)$ is homeomorphic to $(2 k-1)\left(S^{2} \times S^{2}\right)$ if $m$ is odd and homeomorphic to $(2 k-1)\left(\mathbb{C P}^{2} \# \overline{\mathbb{C P}}^{2}\right)$ if $m$ is even.

If $L$ and $L^{\prime}$ are Seiberg-Witten basic classes of $M_{k, n}^{1,1}(m)$, then $\left(L-L^{\prime}\right)^{2}$ is either 0 or $32 k$ by (7). Hence $\left(L-L^{\prime}\right)^{2}$ is never -4 and we can deduce that every $M_{k, n}^{1,1}(m)$ is irreducible. This concludes the proof of Theorem 2 ,

Remark 7. We should point out that the torus surgeries in (21) and (6) are not the only ones we could have chosen. We have verified that many other combinations of surgeries work just as well and give rise to alternative families of exotic smooth structures.

Remark 8. Recall that $M_{1}^{1}$ in [5] contains genus 2 surfaces $q\left(\Sigma_{2} \times\{\mathrm{pt}\}\right)$ with selfintersection 0 . To obtain an alternative construction of exotic smooth structures on $(2 k-1)\left(S^{2} \times S^{2}\right)$ for $k \geq 2$, we can fiber sum $M_{1}^{1}$ with $k-1$ copies of $\Sigma_{2} \times T^{2}$, along genus 2 surfaces $q\left(\Sigma_{2} \times\{\mathrm{pt}\}\right)$ and $\Sigma_{2} \times\left\{\mathrm{pt}^{\prime}\right\}$, and then perform $4(k-1)$ Luttinger surgeries, 4 Luttinger surgeries in each copy of $\Sigma_{2} \times T^{2}$ (cf. 4, 1,). Details of this and a few other similar constructions will appear in a later version. 


\section{ACKNOWLEDGMENTS}

The first author was partially supported by an NSF grant. The second author was partially supported by an NSERC discovery grant. The authors thank Robert E. Gompf for helpful discussions.

\section{REFERENCES}

[1] A. Akhmedov, R. İ. Baykur and B. D. Park, Constructing infinitely many smooth structures on small 4-manifolds, J. Topol. 1 (2008), 409-428.

[2] A. Akhmedov and B. D. Park, New symplectic 4-manifolds with nonnegative signature, J. Gökova Geom. Topol. GGT 2 (2008), 1-13.

[3] A. Akhmedov and B. D. Park, Geography of simply connected spin symplectic 4-manifolds, Math. Res. Lett. 17 (2010), 483-492.

[4] A. Akhmedov and B. D. Park, Exotic smooth structures on small 4-manifolds with odd signatures, Invent. Math. (to appear).

[5] A. Akhmedov and B. D. Park, Exotic smooth structures on $S^{2} \times S^{2}$, arXiv:1005.3346

[6] D. Auroux, S. K. Donaldson and L. Katzarkov, Luttinger surgery along Lagrangian tori and non-isotopy for singular symplectic plane curves, Math. Ann. 326 (2003), 185-203.

[7] R. Fintushel, B. D. Park and R. J. Stern, Reverse engineering small 4-manifolds, Algebr. Geom. Topol. 7 (2007), 2103-2116.

[8] M. H. Freedman, The topology of four-dimensional manifolds, J. Differential Geom. 17 (1982), 357-453.

[9] K. M. Luttinger, Lagrangian tori in $\mathbb{R}^{4}$, J. Differential Geom. 42 (1995), 220-228.

[10] M. Mendes Lopes and R. Pardini, On surfaces with $p_{g}=2 q-3$, arXiv:0811.0390

[11] B. D. Park, A gluing formula for the Seiberg-Witten invariant along $T^{3}$, Michigan Math. J. 50 (2002), 593-611.

[12] M. Symington, Symplectic rational blowdowns, J. Differential Geom. 50 (1998), 505-518.

[13] C. H. Taubes, The Seiberg-Witten invariants and symplectic forms, Math. Res. Lett. 1 (1994), 809-822.

School of Mathematics, University of Minnesota, Minneapolis, MN, 55455, USA

E-mail address: akhmedov@math.umn.edu

Department of Pure Mathematics, University of Waterloo, Waterloo, On, N2L 3G1, CANADA

E-mail address: bdpark@math.uwaterloo.ca 